\title{
Anti-inflammatory activity of Radix Angelicae biseratae in the treatment of osteoarthritis determined by systematic pharmacology and in vitro experiments
}

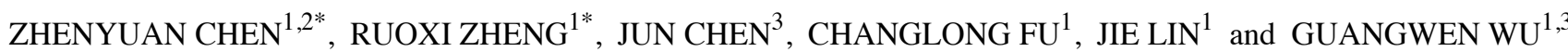 \\ ${ }^{1}$ Osteopathy Institute of Integrative Medicine, Academy of Integrative Medicine, Fujian University of \\ Traditional Chinese Medicine, Fuzhou, Fujian 350122; ${ }^{2}$ Department of Clinical Medicine, Fujian Health College, \\ Fuzhou, Fujian 350101; ${ }^{3}$ Fujian Provincial Key Laboratory of Integrative Medicine on Geriatrics, \\ Fujian University of Traditional Chinese Medicine, Fuzhou, Fujian 350122, P.R. China
}

Received March 11, 2020; Accepted September 2, 2020

DOI: $10.3892 /$ etm.2020.9437

\begin{abstract}
Radix Angelicae biseratae is a widely used Chinese traditional herbal medicine for osteoarthritis (OA). Its therapeutic efficacy has been confirmed in clinical practice. However, its mechanisms of action in treating OA have remained elusive. The purpose of the present study was to identify active components with good oral bioavailability and drug-like properties from Radix Angelicae biseratae through systematic pharmacology and in vitro experiments to determine targets of Radix Angelicae biseratae in the treatment of OA. The functional components of Radix Angelicae biseratae were screened from the Traditional Chinese Medicine Systems Pharmacology database based on oral bioavailability and drug-like properties. Subsequently, the databases STITCH, Open Targets Platform and DrugBank were searched and microarray analysis was performed to screen the active components of Radix Angelicae biseratae to treat $\mathrm{OA}$ and predict its potential target proteins. The interaction network and protein interaction network were then generated and examined, molecular docking between active components and targets was performed and the enrichment of potential target proteins was analyzed. Finally, reverse transcription-quantitative (RT-q)PCR and western blot analyses were used to verify the therapeutic effect of Radix Angelicae biseratae extract on the expression of OA-associated target
\end{abstract}

Correspondence to: Dr Guangwen Wu, Osteopathy Institute of Integrative Medicine, Academy of Integrative Medicine, Fujian University of Traditional Chinese Medicine, 1 Qiuyang Road, Fuzhou, Fujian 350122, P.R. China

E-mail: wuguangwenfj@163.com

*Contributed equally

Key words: Radix Angelicae biseratae, osteoarthritis, antiinflammation, molecular mechanism, systematic pharmacology, traditional Chinese medicine proteins. The results provided eight active components in Radix Angelicae biseratae, which were firmly linked to 20 targets of OA. In combination with molecular docking and the analysis of the interaction network between components and targets, it was suggested that sitosterol was a major active component of Radix Angelicae biseratae in the treatment of OA. Protein interaction network analysis suggested that prostaglandin-endoperoxide synthase 2 (PTGS2), nitric oxide synthase 3 and cytochrome P450 2B6 may be critical targets for Radix Angelicae biseratae in the treatment of OA. In addition, RT-qPCR and western blot analyses suggested that Radix Angelicae biseratae extract inhibited the mRNA and protein expression of PTGS2 in degenerative articular cartilage cells in vitro, whilst other targets remain to be verified. Functional enrichment analysis indicated that Radix Angelicae biseratae confers pharmacological efficacy towards OA through exerting anti-inflammatory effects and immune regulation.

\section{Introduction}

Osteoarthritis (OA) is a complex chronic, severe degenerative arthropathy involving the synovium of the joint. It has a significant economic impact on the public health sector and as the population ages, the number of patients is expected to increase (1). OA is widely recognized as an 'arthralgia syndrome' in Traditional Chinese Medicine (TCM). Inflammation is a critical process in the occurrence and development of $\mathrm{OA}$, as it is able to stimulate and enhance the catabolism of chondrocytes and lead to joint injury. One of the primary symptoms of OA is pain, which may be caused and worsened by inflammation. Inhibition of inflammation is able to provide an effective treatment for OA by relieving pain and reducing joint injury (2-4).

Radix Angelicae biseratae is the root of the plant Angelica biserrata, known as 'Duhuo' in China. As a natural herb, Radix Angelicae biseratae has long been used to treat 'arthralgia syndrome' by eliminating dampness and relieving pain according to the concepts of TCM (5). Modern pharmacological studies have demonstrated that Radix Angelicae 
biseratae has a significant anti-inflammatory effect for the treatment of OA (6). However, the chemical composition of Radix Angelicae biseratae is complex and the mechanism for treating OA at the molecular level has remained elusive.

Systematic pharmacology is a subject where the knowledge of systems biology is applied to pharmacology. The purpose of this discipline is to clarify how drugs act on the body through biological systems. Instead of evaluating the effect of a drug by analyzing a particular protein-drug interaction, systematic pharmacology suggests that drugs act by forming a network of interactions (7). Interaction systems may include drug-protein and protein-protein interactions, as well as genetic, signaling and physiological interactions (7). Systematic pharmacology uses bioinformatics and statistics to integrate and explain the network of drug interactions, which provides an effective method to investigate the underlying mechanisms of TCM and other naturally occurring compounds for the treatment of a number of diseases $(8,9)$.

Therefore, in the present study, systematic pharmacology was used to screen the potential active components of Radix Angelicae biseratae for the treatment of OA, to predict and verify its targets and provide a theoretical basis for drug development and disease treatment. An experimental flowchart for the present study is presented in Fig. 1.

\section{Materials and methods}

Screening for active components. The TCM systems pharmacology (TCMSP) database (http://lsp.nwu.edu.cn/tcmsp. php) is a systematic pharmacological database for evaluating the pharmacokinetic properties of TCM or associated components (10). It provides data on the absorption, distribution, metabolism and systemic excretion (ADME) of drugs with potential biological effects, such as oral bioavailability (OB) and druglikeness (DL), as well as Caco-2 and blood-brain barrier permeability. OB is the most critical feature of oral drugs because it is important in evaluating the effectiveness of drug distribution through the systemic circulation (11). DL refers to the similarity between components and known drugs. In drug development, DL evaluation helps to identify qualified components and improve the success rate of candidate drugs (12). Based on the published literature and preliminary information in the TCMSP database (13), the drug name 'Radix Angelicae biseratae' was entered into the search window in the TCMSP database. The ingredients with OB $\geq 30 \%$ and $\mathrm{DL} \geq 18 \%$ were screened for further study.

Identification of drug targets. The STITCH (http://stitch.embl.de/) database was used to identify the potential targets of active components in Radix Angelicae biseratae (14). STITCH is a database used to explore and predict the interaction between components and proteins. Through experiments, databases and literature evidence, chemicals associated with other chemicals and proteins were retrieved. In the present study, the active components were inputted into the STITCH database and human species were set up to screen the target proteins of Radix Angelicae biseratae with a confidence score $>0.4$ for further analysis.

Identification of disease targets. OA targets were collected from the Open Targets platform (https://www.targetvalidation. org) $(15,16)$ and the DrugBank database (https://www. drugbank.ca) (17-19). The Open Targets platform is a comprehensive and robust data integration platform for accessing and visualizing potential drug targets related to diseases. It brings together a variety of data types designed to help users identify and prioritize targets for further research (20). The disease name 'osteoarthritis' was entered into the search window in the Open Targets platform database and target proteins were collected for further analysis. DrugBank database is a comprehensive online database that contains information concerning drugs and drug targets. The DrugBank database was searched with the key word 'osteoarthritis' to screen and collect targets for known drugs in the treatment of OA. Subsequently, the proteins overlapping the Radix Angelicae biseratae targets were selected for further study.

Construction of component-target network. A 'component-target network' diagram was constructed using Cytoscape 3.7 .2 (https://cytoscape.org/) software in order to reveal the major active components and regulatory mechanisms of Radix Angelicae biseratae in the treatment of OA (21).

Construction of target-target interaction network. In order to identify the critical proteins among the target proteins identified as specified above, a 'target-target interaction network' was established using the Search Tool for the Retrieval of Interacting Genes and proteins (STRING; v11.0; http://string-db.org/) and visualized with Cytoscape 3.7 .2 (22).

Gene functional enrichment analysis. ClusterProfiler was used to analyze the enrichment of gene function and signaling pathways. ClusterProfiler is an $\mathrm{R}$ package for gene cluster enrichment analysis, which may be used to understand the function of genes and the enrichment of pathways (23). When provided with the overlapping target gene list, ClusterProfiler uses the Gene Ontology (GO) database to analyze the biological function and signaling pathway enrichment of these genes $(24,25)$.

Verification of target proteins by $O A$ gene expression array. A set of microarray data of gene expression in cartilage tissue of patients with OA was obtained from the gene expression omnibus (GEO) database (https://www.ncbi.nlm. nih.gov/geo/; dataset no. GSE75181). The dataset contained 48 samples, of which 12 non-control samples and 12 control samples treated with IL-1 $\beta$ were selected. The gene matrix file of the GSE75181 dataset was downloaded and it was standardized with the limma package (26), including background correction, standardization and normalization. The impute package was used to fill in the missing values (27). The platform annotation file was used to annotate the probes and delete those probes (genesymbols) that did not match the gene. For multiple probes targeting the same gene, the average value of different probes was used as the expression value of the gene. Depending on whether the samples were treated with IL-1 $\beta$ or not, the samples were divided into two groups: The non-control group and the control group. Regarding the difference in expression, the P-value and fold change (FC) in expression of the non-control vs. control samples were calculated using the limma package. Adjusted 


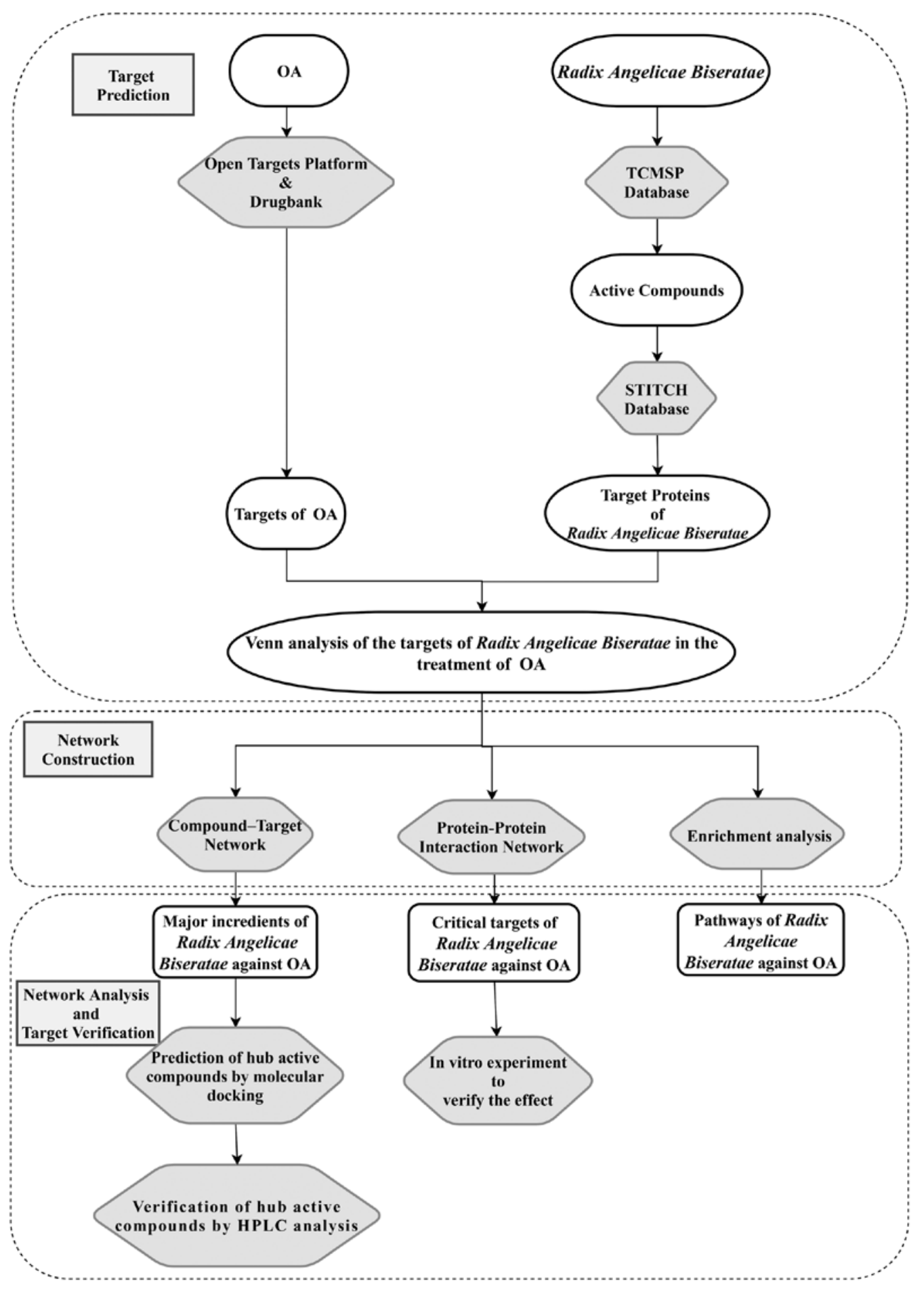

Figure 1. Flowchart of the experimental procedures. OA, osteoarthritis; TCMSP, Traditional Chinese Medicine Systems Pharmacology.

P-value (adj.P.Val) $<0.05$ and $\mid \log 2 \mathrm{FCl}>1$ were chosen as the threshold for filtering the significant differentially expressed mRNA and display the results in the form of volcano plot. Critical proteins on the volcano plot were then marked.

Prediction of hub target protein-component docking. The Blind Docking server (https://bio-hpc.ucam. edu/achilles/entry) was used for molecular docking experiments (28). The crystal structure of active components was downloaded from the PubChem database (https://pubchem. ncbi.nlm.nih.gov) and the structure of the hub target proteins was obtained from the protein databank (PDB; https://rscb.org) (29). BIOVIA DiscoveryStudio ${ }^{\circledR} 2016$ software (Dassault Systemes) was used to modify the protein structure for molecular docking. All water molecules and ligands were removed and the necessary hydrogen atoms were added. The obtained receptor and ligand structures were uploaded to the Blind Docking server for docking calculation and the best structures with the lowest binding energy were used. 
In vitro verification

Animals. Male 4-week-old Sprague Dawley rats (n=6; weight, 80-100 g) were purchased from Shanghai SLAC Laboratory Animal Co., Ltd [Laboratory Animal Use Certificate no. SCXK(SH)2017-0005]. The present study was approved by the Animal Care and Use Committee of Fujian University of Traditional Chinese Medicine (Fuzhou, China). Experiments involving animals complied with the 2006 Edition of the Guidelines for the Care and Use of Experimental Animals by the Ministry of Science and Technology, China (30).

Preparation and analysis of Radix Angelicae biseratae extract. Radix Angelicae biseratae was purchased from the local Guo Yi Tang Chinese herbal medicine store. The original herb was identified as Radix Angelicae biseratae by Dr Wen $\mathrm{Xu}$ at the Department of Pharmacology, Fujian University of Traditional Chinese Medicine (Fujian, China). Radix Angelicae biseratae (500 g) was crushed into a fine powder and boiled twice with 41 of $80 \%$ ethanol for $1 \mathrm{~h}$. The ethanolic extract was collected and filtered. The filtrate was concentrated to $500 \mathrm{ml}$ at $50^{\circ} \mathrm{C}$ under reduced pressure and the concentration was $1 \mathrm{~g} / \mathrm{ml}$. The content of sitosterol in the extract was determined by high-performance liquid chromatography (HPLC) method using an Agilent 1200 HPLC system (Agilent Technologies, Inc.) and the Thermo Scientific ${ }^{\mathrm{TM}}$ BETASIL $^{\mathrm{TM}}$ C18 column (4.6x150 mm, $5 \mu \mathrm{m}$; Thermo Fisher Scientific, Inc.). The column temperature was set at $35^{\circ} \mathrm{C}$ and the injection volume was $10 \mu \mathrm{l}$. The mobile phase was $100 \%$ methanol and the flow rate was $1.0 \mathrm{ml} / \mathrm{min}$. Ultraviolet detection wavelengths were set at $210 \mathrm{~nm}$ at 0-30 $\mathrm{min}$.

Chondrocyte observation and identification. Chondrocytes were isolated from the knee articular cartilage of 4-week-old Sprague Dawley rats. After the rats were anesthetized by intraperitoneal injection of $30 \mathrm{mg} / \mathrm{kg}$ pentobarbital, they were sacrificed via rapid decapitation. The chondrocytes were isolated, cultured and verified, as previously described $(31,32)$.

Degenerative chondrocyte model. The degenerative chondrocyte model was established as previously described $(33,34)$. In brief, the chondrocytes at passage three were exposed to $10 \mathrm{ng} / \mathrm{ml} \mathrm{IL-1} \beta$ for $24 \mathrm{~h}$.

Experimental grouping. Cells were divided into the following groups: i) control group without treatment; ii) degenerative chondrocyte group; iii) degenerative chondrocytes with $1 \mu \mathrm{M}$ Radix Angelicae biseratae extract group; iv) degenerative chondrocytes with $5 \mu \mathrm{M}$ Radix Angelicae biseratae extract group; and v) degenerative chondrocytes with $25 \mu \mathrm{M}$ Radix Angelicae biseratae extract group. The intervention time for all groups was $24 \mathrm{~h}$.

Reverse transcription-quantitative PCR (RT-qPCR). After $24 \mathrm{~h}$ of intervention, total RNA was extracted from chondrocytes of each group using a TRIzol ${ }^{\circledR}$ kit (Invitrogen; Thermo Fisher Scientific, Inc.). RNA(1 $\mu \mathrm{g})$ was reverse transcribed into cDNA using the PrimeScript ${ }^{\mathrm{TM}}$ RT reagent kit (cat. no. RR0047A; Takara Bio, Inc.) in the PCR amplifier (S1000 Thermal Cycler; Bio-Rad Laboratories, Inc.) according to the manufacturer's protocol. The following primer sequences were used: Prostaglandin-endoperoxide synthase 2 (PTGS2) forward, 5'-AGCACAATAGACGCC CAAGA-3' and reverse, 5'-GGAGTCAAAGCATAGGTC TTCA-3'; $\beta$-actin forward, 5'-ACCACTGGCATTGTGATG GA-3' and reverse, 5'-CGCTCGGTCAGGATCTTCT-3'. $\beta$-actin was used to normalize the expression of mRNA. AceQ qPCR SYBR ${ }^{\circledR}$ Green Master Mix (cat. no. Q111-02; Vazyme Biotech Co., Ltd.) was used to detect the respective mRNA expression levels. qPCR was conducted using the 7500 Fast Real-Time PCR system (Applied Biosystems; Thermo Fisher Scientific, Inc.) using the following reaction conditions: Initial denaturation at $95^{\circ} \mathrm{C}$ for $3 \mathrm{~min}$, followed by 40 cycles at $95^{\circ} \mathrm{C}$ for $10 \mathrm{sec}$ and $60^{\circ} \mathrm{C}$ for $30 \mathrm{sec}$; dissolution curve at $95^{\circ} \mathrm{C}$ for $15 \mathrm{sec}, 60^{\circ} \mathrm{C}$ for $60 \mathrm{sec}$ and $95^{\circ} \mathrm{C}$ for $15 \mathrm{sec}$. The experiment was performed three times and the gene expression ratios were expressed in the form of average value and standard deviation according to the independent measurement results of the three experiments. The $2^{-\Delta \Delta \mathrm{Cq}}$ method (35) was used to evaluate the experimental results.

Western blot analysis. The expression levels of the target proteins in chondrocytes of each group were measured by western blot analysis. Western blotting and semi-quantitative analysis were performed according to a previously published protocol (36). The following antibodies were used: PTGS2 antibody (1:1,000 dilution; cat. no. ab52237; Abcam), $\beta$-actin antibody (1:2,000 dilution; cat. no. ab8227; Abcam) and horseradish peroxidase-conjugated secondary antibodies $(1: 20,000$ dilution; cat. no. AP132P; Merck KGaA).

Statistical analysis. The experimental data were processed and analyzed by Graph Pad Prism 8.02 software (GraphPad Software, Inc.). The Shapiro-Wilk test was used to determine the normality of all groups of data. If the data exhibited a normal distribution, they were compared by one-way analysis of variance followed by a least significant difference or Games Howell post-hoc test; if not, the Kruskal-Wallis test was used and the Mann-Whitney U test with Bonferroni's correction was applied as a post hoc test. $\mathrm{P}<0.05$ was considered to indicate a statistically significant difference.

\section{Results}

Components and potential targets for Radix Angelicae biseratae. The TCMSP database was searched with the key words 'Radix Angelicae biseratae' for 99 herbal ingredients. When applying the threshold specified in the methods section, nine herbal ingredients were obtained.

These nine components screened from the TCMSP database were searched in the STITCH database, of which eight components had 30 target proteins. Among them, ammidin had five targets, isoimperatorin had seven targets, sitosterol had 14 targets, O-acetyl columbianetin had nine targets, angelol D had two targets, angelol $\mathrm{G}$ had one target, angelicone had five targets and nodakenin had eight targets. The ADME properties of these eight components are presented in Table I.

Identification of OA-associated proteins. By searching the Open Targets Platform and DrugBank database, 20 targets 
A

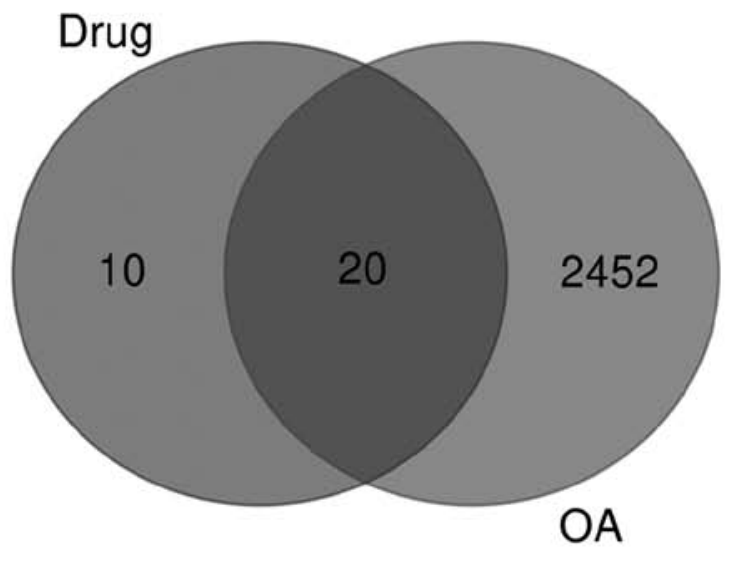

B

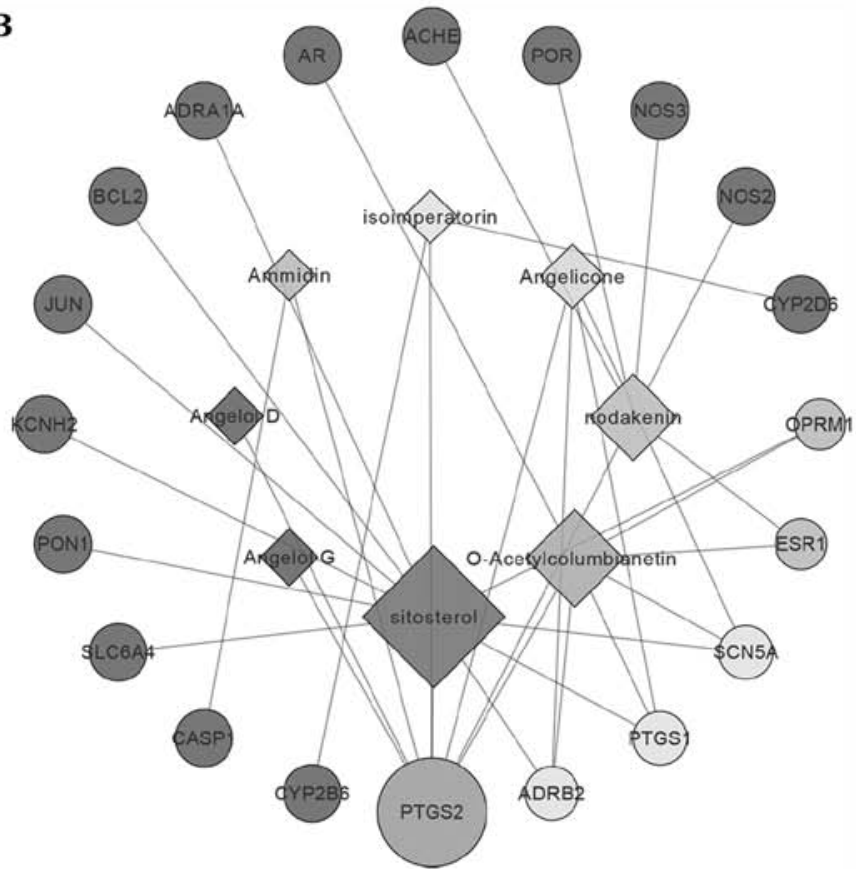

Figure 2. Venn analysis and network analysis of active components and potential targets of Radix Angelicae biseratae in the treatment of OA. (A) Venn diagram of target proteins of Radix Angelicae biseratae and OA-related proteins based on the Open Targets Platform and Drugbank database. (B) The compound-target network of Radix Angelicae biseratae to treat OA, the square nodes represent the compounds, the circular nodes represent the targets, and the node size is proportional to the degree. OA, osteoarthritis; PTGS2, prostaglandin-endoperoxide synthase 2; NOS3, nitric oxide synthase 3; CYP2B6, cytochrome P450 2B6; ACHE, acetylcholinesterase; ESR1, estrogen receptor 1; SLC6A4, solute carrier family 6 member 4; CYP2D6, cytochrome P450 2D6; NOS2, nitric oxide synthase 2; OPRM1, opioid receptor $\mu 1$; CASP1, caspase 1; AR, androgen receptor; PTGS1, prostaglandin-endoperoxide synthase 1; PON1, paraoxonase 1; ADRA1A, adrenoceptor $\alpha 1 \mathrm{~A}$; POR, cytochrome P450 oxidoreductase; ADRB2, adrenoceptor $\beta 2$; KCNH2, potassium voltage-gated channel subfamily $\mathrm{H}$ member 2; BCL2, B-cell lymphoma 2; SCN5A, sodium voltage-gated channel $\alpha$ subunit 5.

Table I. Absorption, distribution, metabolism and excretion properties of 8 active components from Radix Angelicae biseratae.

\begin{tabular}{llcl}
\hline Mol ID & \multicolumn{1}{c}{ Molecule name } & OB & DL \\
\hline MOL001941 & Ammidin & 34.55 & 0.28 \\
MOL001942 & Isoimperatorin & 45.46 & 0.27 \\
MOL000358 & Sitosterol & 36.91 & 0.23 \\
MOL003608 & O-Acetylcolumbianetin & 60.04 & 0.29 \\
MOL004777 & Angelol D & 34.85 & 0.30 \\
MOL004778 & Angelol G & 46.03 & 0.29 \\
MOL004780 & Angelicone & 30.99 & 0.26 \\
MOL004792 & Nodakenin & 57.12 & 0.27 \\
\hline
\end{tabular}

OB, oral bioavailability; DL, druglikeness.

linked to the treatment of OA were screened (Fig. 2A). As presented in Fig. 2B, a network of pharmacologically active components and targets was established. Of the eight components, seven had OA-related targets. Among these seven components, sitosterol (degree=11) may have an essential role in the treatment of OA, followed by O-acetylcolumbianetin (degree $=7$ ) and nodakenin (degree=6). All components were linked to PTGS2, which suggests that Radix Angelicae biseratae may be a potential inhibitor of PTGS2.
Target-target interaction analysis. Using Cytoscape 3.7.1, a diagram of the 'target-target network' was constructed based on the query results of the STRING database (Fig. 3A). In addition, the bar plot was constructed according to the degrees of connection between the targets (Fig. 3B). The results indicated that nitric oxide synthase 3 (NOS3), PTGS2 and cytochrome P450 2B6 (CYP2B6) were the hub targets in this network with high degree values.

Pathway analysis. The ClusterProfiler package in $\mathrm{R}$ was used to perform GO analysis of the functional characteristics of targets and the threshold was set as $\mathrm{P} \leq 0.01$. The results of the GO analysis suggested that 75 GO terms were enriched in 'Biological Process'. The first 20 items with the most significant P-values are provided in Fig. 4. The neurotransmitter metabolic process, response to nutrient levels and nitric oxide metabolic process were among the functional roles of Radix Angelicae biseratae and this may explain its anti-inflammatory action.

Verification of the target protein in GSE75181. A total of 665 differentially expressed genes were identified from two groups of cartilage samples. As presented in Fig. 5, compared with the hub target of Radix Angelicae biseratae, PTGS2 was significantly upregulated in the IL-1 $\beta$-treated group (adj.P.Val $<0.05 ; \mid \log 2 \mathrm{FCl}>1)$.

Docking of hub target proteins and major components. Docking studies were performed using the Blind Docking server in the active sites of the two critical targets to investigate 

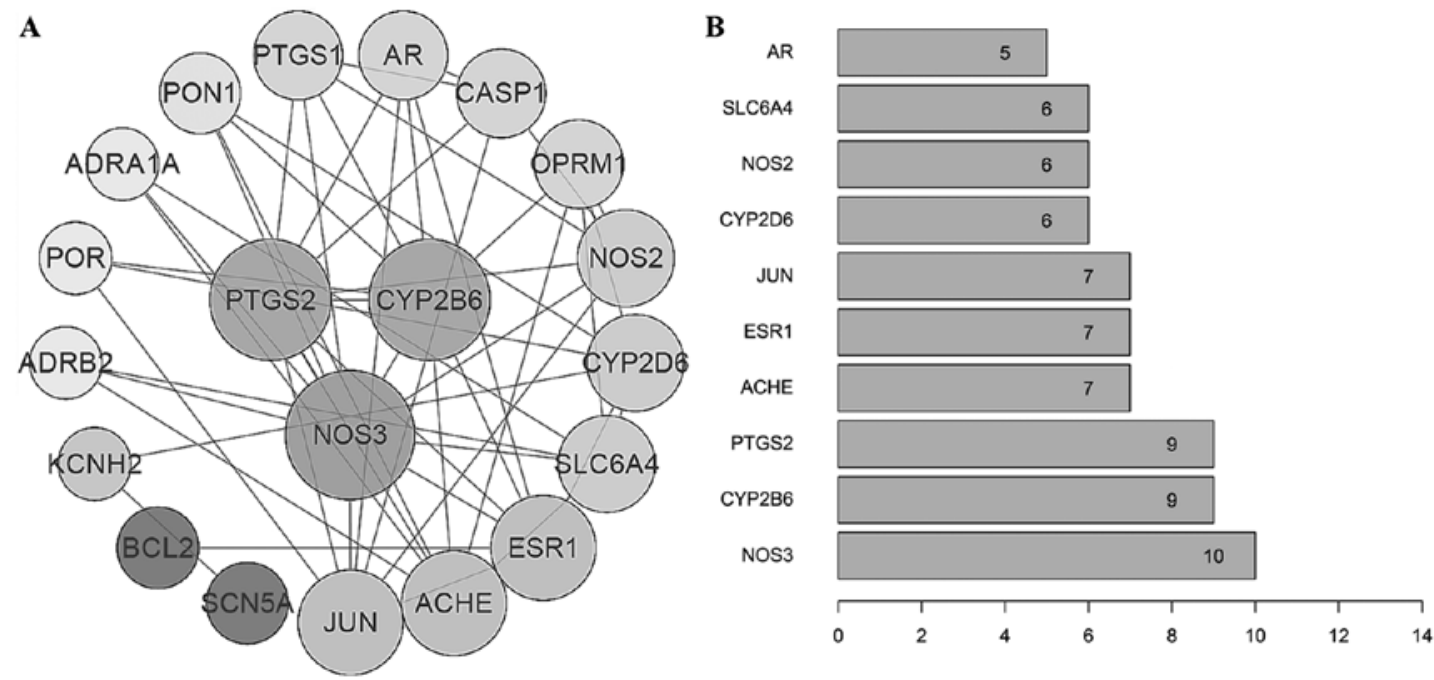

Figure 3. 'Target-target' interaction network analysis. (A) Target-target interaction network constructed using Cytoscape. The sizes of the nodes are proportional to their degrees. (B) Barplot of the degrees of connection between the targets. PTGS2, prostaglandin-endoperoxide synthase 2; NOS3, nitric oxide synthase 3; CYP2B6, cytochrome P450 2B6; JUN, jun proto-oncogene; ACHE, acetylcholinesterase; ESR1, estrogen receptor 1; SLC6A4, solute carrier family 6 member 4; CYP2D6, cytochrome P450 2D6; NOS2, nitric oxide synthase 2; OPRM1, opioid receptor $\mu 1$; CASP1, caspase 1; AR, androgen receptor; PTGS1, prostaglandin-endoperoxide synthase 1; PON1, paraoxonase 1; ADRA1A, adrenoceptor $\alpha 1 \mathrm{~A}$; POR, cytochrome P450 oxidoreductase; ADRB2, adrenoceptor $\beta 2 ; \mathrm{KCNH} 2$, potassium voltage-gated channel subfamily $\mathrm{H}$ member 2; BCL2, B-cell lymphoma 2; SCN5A, sodium voltage-gated channel $\alpha$ subunit 5 .

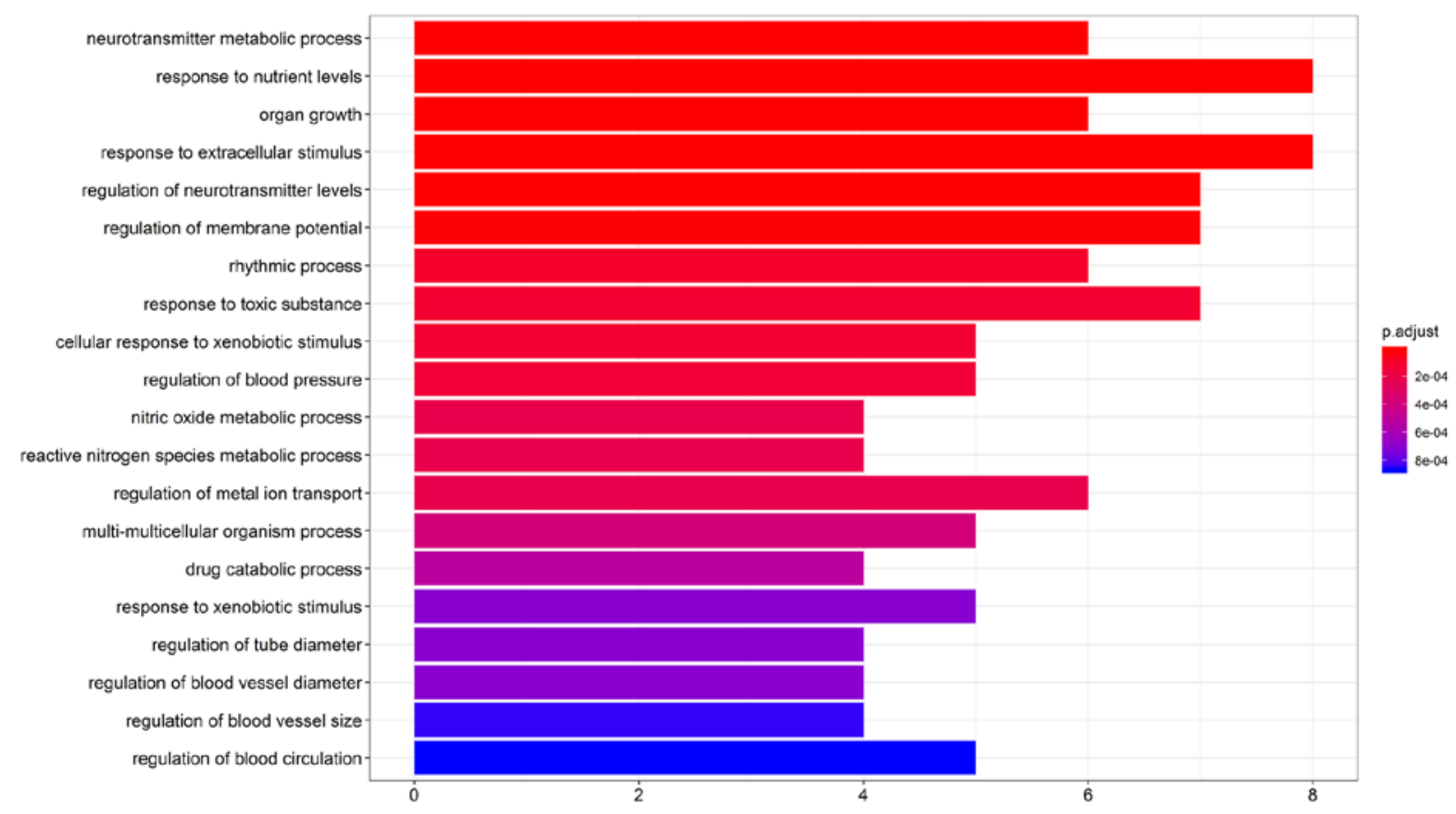

Figure 4. Top 20 biological process terms from the Gene Ontology analysis. The names of the path are specified on the vertical axis, while the horizontal axis represents the ratio of the number of enriched genes to the total number of uploaded genes. The color of the bars indicates the significance of the P-values.

the possible interactions between the active components and potential targets, namely PTGS2 (PDB ID: 5F19) and NOS3 (PDB ID: 3NOS). In general, a lower binding energy indicates a stronger binding between the molecules (37). The conformation with the lowest binding energy was selected in the component-target complex between each component and the target and their binding energy is listed in Table II.

As presented in Table II, among the active components, a certain conformation of the complex of sitosterol and hub targets had the highest binding energy. Discovery Studio was used to map the binding pattern of sitosterol to hub target proteins. The binding pattern of sitosterol in the active site of PTGS2 is presented as a three-dimensional model in Fig. 6A and a two-dimensional diagram is shown in Fig. 6B. Sitosterol was indicated to interact with the ASN537 residue in the $B$ chain and also, the GLY533 residue in the B chain has two hydrogen bonds, and sitosterol has two hydrophobic interactions with the PHE142 and LEU145 amino acid residues. The binding pattern of sitosterol at the active site of NOS3 is presented in its three-dimensional model in Fig. 6C and a 


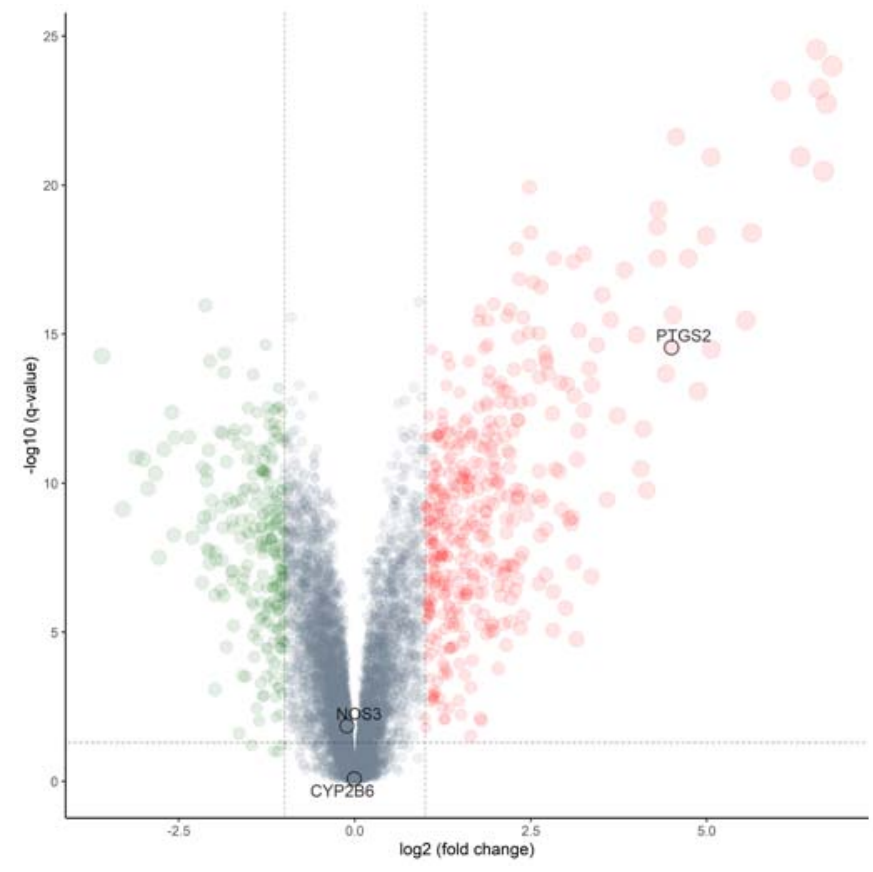

Figure 5. Volcano plot of all genes. The red dots indicate the upregulated genes (adj.P.Val $<0.05$ and $\log F C>1$ ), the green dots indicate the significantly downregulated genes (adj.P.Val $<0.05$ and $\log \mathrm{FC}<-1$ ) and the gray dots indicate the genes with no obvious change. PTGS2, prostaglandin-endoperoxide synthase 2; NOS3, nitric oxide synthase 3; CYP2B6, cytochrome P450 2B6; FC, fold change; adj.P.Val, adjusted P-value.

two-dimensional diagram is provided in Fig. 6D. Sitosterol exhibited an electrostatic interaction in the TRP356 residue on the B chain, and also involved in the alkyl-alkyl interaction with ALA423, MET358, VAL185, VAL418, CYS184, PHE353, ARG183, MET339, PHE473, ALA181, TRP178 and PRO334 in the A chain. These interactions increased the binding affinity between the two molecules.

Analysis of active components of Radix Angelicae biseratae extract. HPLC analysis indicated that the Radix Angelicae biseratae extract contained sitosterol (Fig. 7A and B), which was demonstrated to be a significant active compound in the treatment of OA that may be responsible for its anti-inflammatory activity, as determined by network analysis and molecular docking.

Radix Angelicae biseratae extract regulates gene and protein expression in the degenerative chondrocyte model. In order to verify the effect of Radix Angelicae biseratae extract on inflammatory factors in degenerative chondrocytes, the rat chondrocytes were treated with $10 \mathrm{ng} / \mathrm{ml} \mathrm{IL-1 \beta}$ for $24 \mathrm{~h}$, following which they were treated with Radix Angelicae biseratae extract $(1,5$ or $25 \mu \mathrm{M})$ for $24 \mathrm{~h}$. As presented in Fig. 8, the expression of PTGS2 in the IL-1 $\beta$ group was significantly higher compared with that in the regular group, which was consistent with the results of the gene expression array. At the same time, Radix Angelicae biseratae extract significantly decreased the expression of PTGS2 in degenerative chondrocytes treated with IL-1 $\beta(\mathrm{P}<0.05)$, which indicated that certain active components in Radix Angelicae biseratae extract were able to inhibit the expression of PTGS2 in degenerative chondrocytes.
Table II. Lowest binding energy between active components of Radix Angelicae biseratae and the active sites of proteins PTGS2 and 3NOS (Protein Databank ID: 5F19 and 3NOS, respectively) determined using the Blind Docking server $(\mathrm{kcal} / \mathrm{mol})$.

\begin{tabular}{lrr}
\hline Compound & PTGS2 & NOS3 \\
\hline Sitosterol & -11.76 & -10.67 \\
O-Acetylcolumbianetin & -9.12 & -8.71 \\
Ammidin & -8.47 & -8.16 \\
Angelicone & -7.71 & -7.91 \\
Angelol D & -8.16 & -7.34 \\
Angelol G & -8.12 & -7.36 \\
Nodakenin & -9.13 & -9.31 \\
Isoimperatorin & -7.62 & -8.19 \\
\hline
\end{tabular}

PTGS2, prostaglandin-endoperoxide synthase 2; NOS3, nitric oxide synthase 3 .

The protein expression of PTGS2 was determined to verify the effect of Radix Angelicae biseratae on degenerative chondrocytes (Fig. 9A and B). Compared with the regular group, the expression of PTGS2 protein in IL-1 $\beta$-induced degenerative chondrocytes was significantly increased $(\mathrm{P}<0.01)$. Compared with that in the degenerative chondrocyte group, the expression of PTGS2 protein in the Radix Angelicae biseratae groups (1,5 and $25 \mu \mathrm{M})$ was significantly inhibited $(\mathrm{P}<0.05)$.

\section{Discussion}

The systematic pharmacology method has been developed for determining molecular biological networks and may be used to discover novel therapeutic effects of drugs from medicinal plants (8). Therefore, it provides a systematic method to expand the application of available drug components in TCM in a variety of complex diseases (9). OA, which is a common disease of chronic arthritis, is associated with painful symptoms and significantly affects the patients' quality of life $(1,2)$. Although Radix Angelicae biseratae has been used as an effective Chinese herbal medicine for the treatment of OA for several centuries, its pharmacological mechanism has remained to be elucidated (5).

In the present study, the active components and potential targets of Radix Angelicae biseratae in the treatment of OA were evaluated based on systematic pharmacology, including ADME system evaluation, network analysis, pathway analysis and molecular docking. A total of eight active components were screened and were indicated to interact with 30 different targets related to OA. According to the analysis of the components-targets network model, sitosterol had the most significant number of target connections (degree $=11$ ), followed by $\mathrm{O}$-acetyl columbianetin (degree=7) and nodakenin (degree=6). Sitosterol has a wide range of biological functions in vivo, including antitumor, antioxidant, antidiabetic and anti-inflammatory activity and roles in reducing gallstone activity (38). Combined with the results of molecular docking 
A

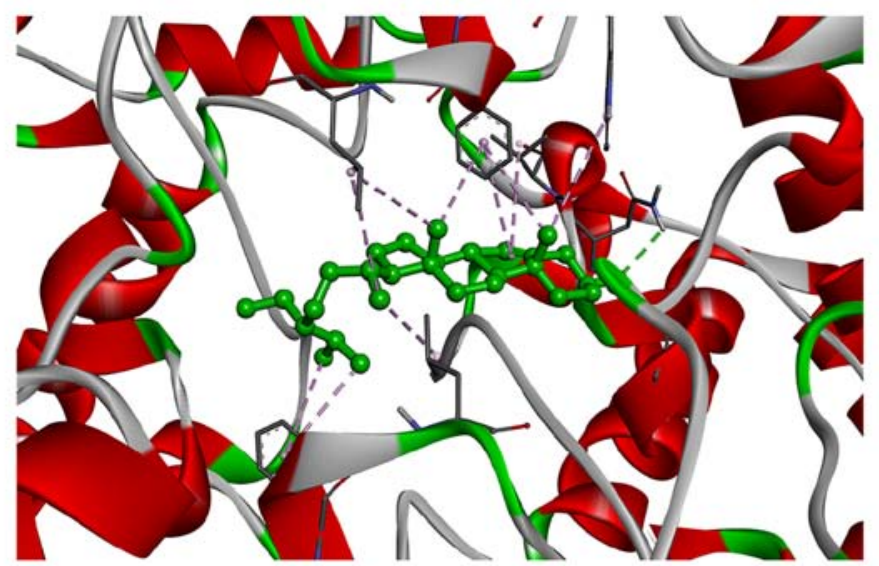

C

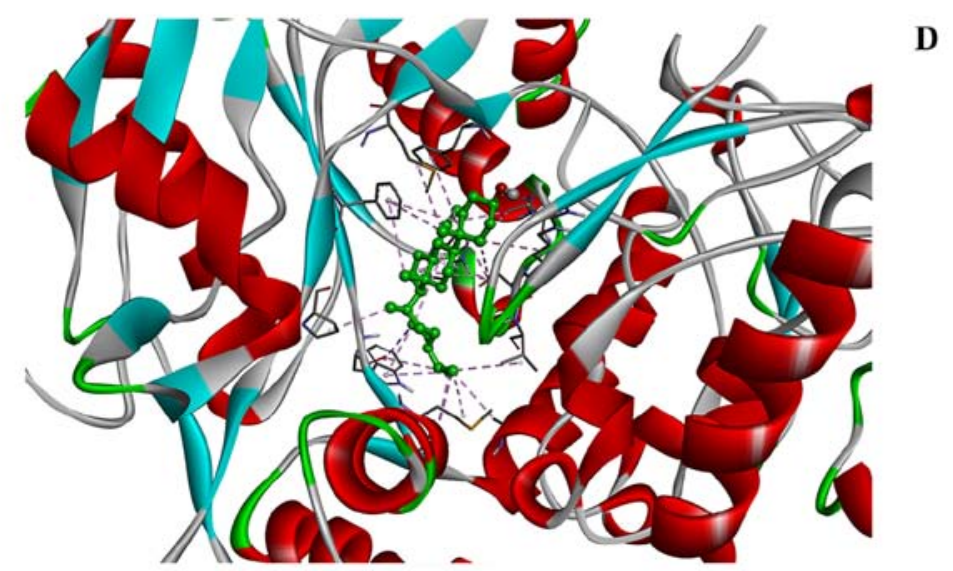

B
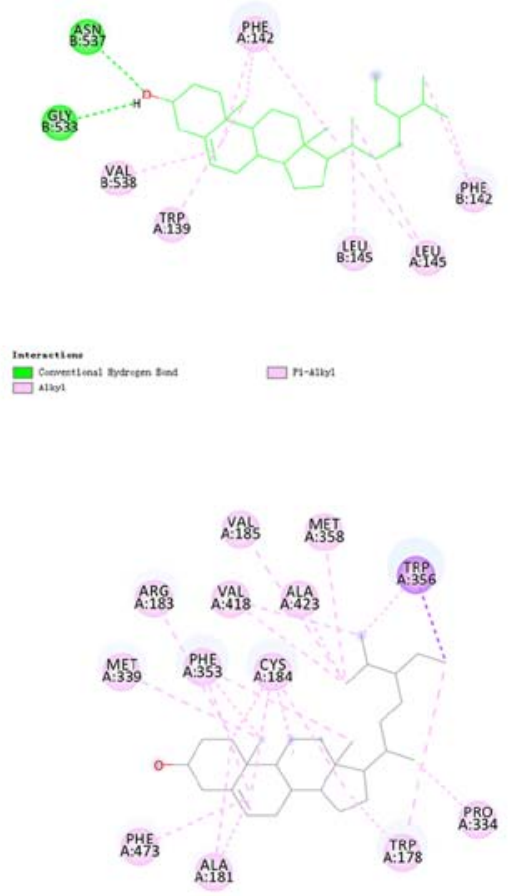

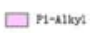

Figure 6. Molecular docking between active components and potential targets. (A) A three-dimensional model of the binding pattern of sitosterol at the active site of the protein PTGS2. Active-site amino acid residues are represented as tubes, while the compound is presented using a stick-ball model. (B) A two-dimensional diagram of Sitosterol at the active site of the protein PTGS2. (C) A three-dimensional model of the binding pattern of Sitosterol at the active site of protein NOS3. (D) A two-dimensional diagram of sitosterol at the active site of the protein NOS3. PTGS2, prostaglandin-endoperoxide synthase 2; NOS3, nitric oxide synthase 3 .
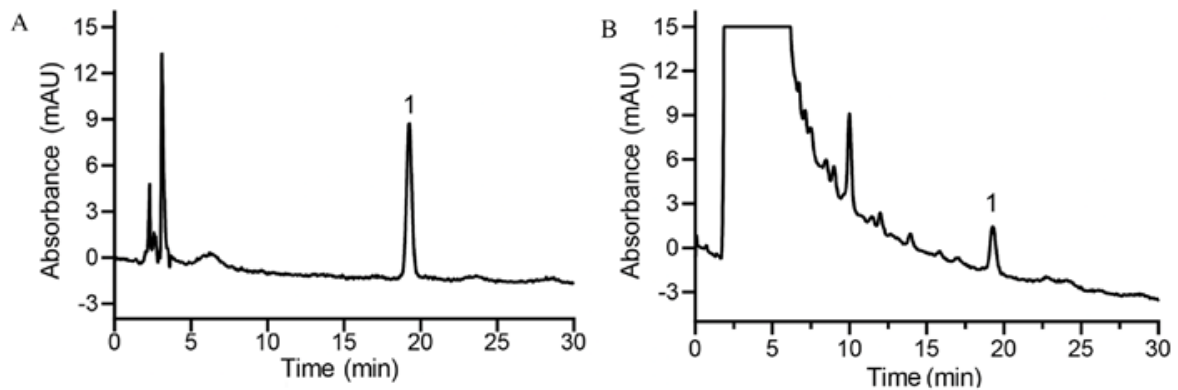

Figure 7. Schematic diagram of HPLC analysis. (A) Chromatogram of sitosterol. (B) Chromatogram of $80 \%$ ethanol extract of Radix Angelicae biseratae. 1, sitosterol; mAU, milli-absorbance units.

in the present study, it is proposed that sitosterol is a critical potential active component of Radix Angelicae biseratae in the treatment of OA.

PTGS2 is the coding gene of cyclooxygenase-2, which is an essential target of OA anti-inflammatory therapy and one of the major inflammatory factors involved in articular cartilage degradation (39). NOS encoded by the NOS3 gene is also a crucial inflammatory mediator in the occurrence and development of OA. It can promote the production of $\mathrm{NO}$ in the microenvironment, increase the production of reactive oxygen species and induce chondrocyte apoptosis (40). CYP2B6 is a member of the cytochrome P450 superfamily of enzymes that is closely related to drug metabolism (41). A recent study demonstrated that CYP2B6 may affect the metabolism of NO under inflammatory stimulation (42). The present study indicated that PTGS2, NOS3 and CYP2B6 are potential targets for Radix Angelicae biseratae in the treatment of OA, with high degree values in the 'target-target' network. Microarray 


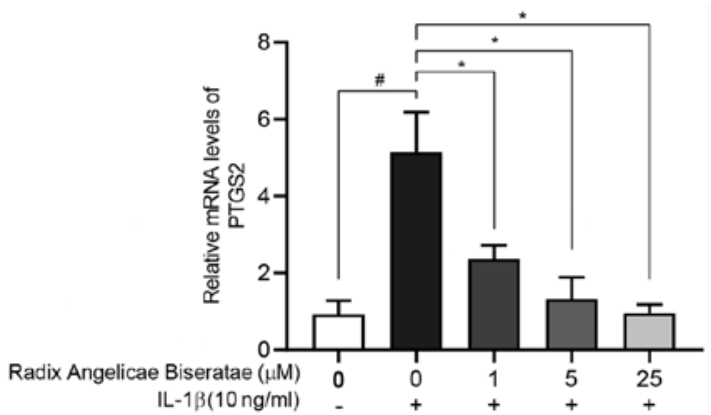

Figure 8. Radix Angelicae biseratae extract inhibits the expression of inflammatory factors in rat chondrocytes induced by IL-1 $\beta$. Chondrocytes were treated with $10 \mathrm{ng} / \mathrm{ml} \mathrm{IL-1} \beta$ for $24 \mathrm{~h}$ and then treated with Radix Angelicae biseratae extract $(1,5$ and $25 \mu \mathrm{M})$ for $24 \mathrm{~h}$. The mRNA levels of PTGS2 in chondrocytes were detected by reverse transcription-quantitative PCR. ${ }^{\#} \mathrm{P}<0.05 ;{ }^{*} \mathrm{P}<0.05$. PTGS2, prostaglandin-endoperoxide synthase 2.
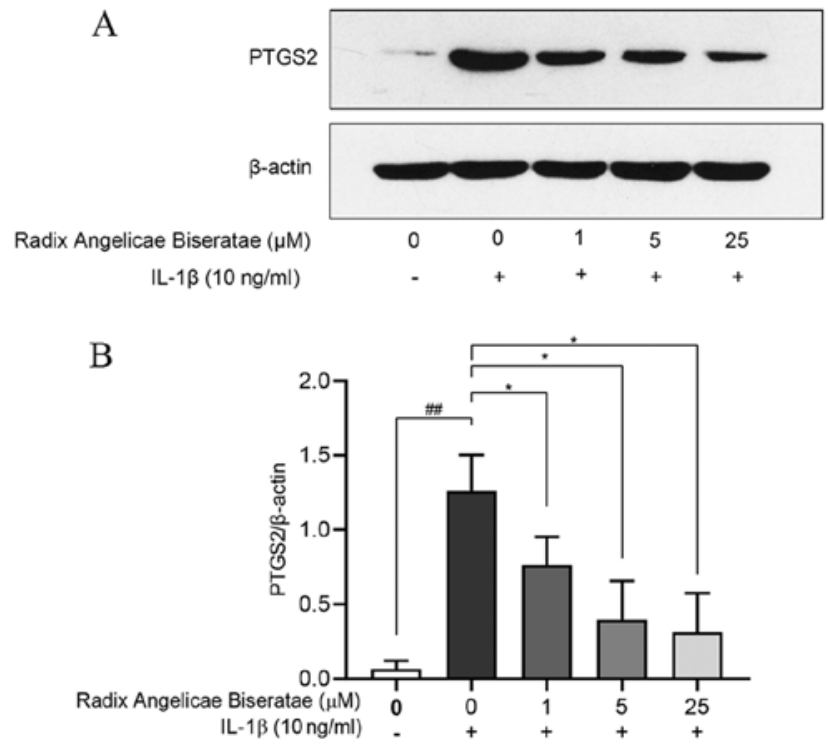

Figure 9. Protein expression of PTGS2 in chondrocytes treated with IL-1 $\beta$ and/or Radix Angelicae biseratae extract. (A) Representative western blot image and (B) quantified expression levels. ${ }^{\#} \mathrm{P}<0.01 ;{ }^{*} \mathrm{P}<0.05$. PTGS2, prostaglandin-endoperoxide synthase 2 .

analysis demonstrated that compared with the non-control group, PTGS2 was highly expressed in the degenerative chondrocyte group. RT-qPCR and western blot analysis confirmed that Radix Angelicae biseratae extract inhibited the expression of PTGS2 in the cartilage degeneration model in vitro, which indicates that certain active components of Radix Angelicae biseratae may have a role in the treatment of OA via anti-inflammatory mechanisms.

Combined with the results of molecular docking, the present results indicated that sitosterol may be a potential inhibitor of NOS3 and PTGS2 and may have an anti-inflammatory effect on OA. The levels of PTGS2 and NOS3 and the effect of sitosterol on their expression in animal models will be investigated in future experiments.

The results of the present pathway analysis indicated that Radix Angelicae biseratae exerted its pharmacological effects in OA by modulating multiple pathways, including drug metabolism, inflammation and immune modulation.
In conclusion, sitosterol was demonstrated to be a critical active component of Radix Angelicae biseratae in the treatment of OA. Radix Angelicae biseratae extract reduced the expression of PTGS2 in degenerative chondrocytes, which may be the underlying mechanism of action of Radix Angelicae biseratae in reducing inflammation in the treatment of OA. However, the present study is based on data mining analysis and in vitro experiments and the results require to be further verified by in vivo experiments.

\section{Acknowledgements}

The authors would like to thank Dr Wen $\mathrm{Xu}$ from the Department of Pharmacology, Fujian University of Traditional Chinese Medicine (Fuzhou, China), for his help with the identification of the original herbs.

\section{Funding}

The present study was supported by the National Natural Science Foundation of China (grant no. 81774345), the Natural Science Foundation of Fujian Province (grant no. 2018J01874) and the Health Family Planning Research Talent Training Project of Fujian Province (grant no. 2017-ZQN-62).

\section{Availability of data and materials}

The datasets used and/or analyzed during the current study are available from the corresponding author on reasonable request.

\section{Authors' contributions}

ZC and GW conceived and designed the study. ZC, RZ, CF, JC and JL determined the active components of Radix Angelicae biseratae and potential targets for the treatment of OA, downloaded datasets and screened differentially expressed genes. $\mathrm{ZC}$ and $\mathrm{RZ}$ performed the experiments and analyzed the data. $\mathrm{ZC}$ ran the statistics and wrote the manuscript. GW reviewed and edited the manuscript. All authors read and approved the final manuscript.

\section{Ethics approval and consent to participate}

All animal experimental procedures were approved by the Animal Care and Use Committee of Fujian University of Traditional Chinese Medicine (Fuzhou, China).

\section{Patient consent for publication}

Not applicable.

\section{Competing interests}

The authors declare that they have no competing interests.

\section{References}

1. Lespasio MJ, Piuzzi NS, Husni ME, Muschler GF, Guarino A and Mont MA: Knee osteoarthritis: A primer. Perm J 21: 16-183, 2017. 
2. Bonnet CS and Walsh DA: Osteoarthritis, angiogenesis and inflammation. Rheumatology (Oxford) 44: 7-16, 2005

3. Shen J, Abu-Amer Y, O'Keefe RJ and McAlinden A: Inflammation and epigenetic regulation in osteoarthritis. Connect Tissue Res 58: 49-63, 2017.

4. Urban $\mathrm{H}$ and Little CB: The role of fat and inflammation in the pathogenesis and management of osteoarthritis. Rheumatology (Oxford) 57 (Suppl 4): iv10-iv21, 2018.

5. Ma J, Huang J, Hua S, Zhang Y, Zhang Y, Li T, Dong L, Gao Q and Fu X: The ethnopharmacology, phytochemistry and pharmacology of Angelica biserrata: A review. J Ethnopharmacol 231: $152-169,2019$.

6. Lyu S, Ji B, Gao W, Chen X, Xie X and Zhou J: Effects of angelicae pubescentis and loranthi decotion on repairing knee joint cartilages in rats. J Orthop Surg Res 12: 189, 2017.

7. Hopkins AL: Network pharmacology: The next paradigm in drug discovery. Nat Chem Biol 4: 682-690, 2008.

8. Zhang YF, Huang Y, Ni YH and Xu ZM: Systematic elucidation of the mechanism of geraniol via network pharmacology. Drug Des Devel Ther 13: 1069-1075, 2019.

9. Yue SJ, Xin LT, Fan YC, Li SJ, Tang YP, Duan JA, Guan HS and Wang CY: Herb pair Danggui-Honghua: Mechanisms underlying blood stasis syndrome by system pharmacology approach. Sci Rep 7: 40318, 2017.

10. Ru J, Li P, Wang J, Zhou W, Li B, Huang C, Li P, Guo Z, Tao W, Yang Y, et al: TCMSP: A database of systems pharmacology for drug discovery from herbal medicines. J Cheminform 6: 13 2014.

11. Cabrera-Pérez $\mathrm{M}$ and Pham-The H: Computational modeling of human oral bioavailability: What will be next? Expert Opin Drug Discov 13: 509-521, 2018.

12. Tao W, Xu X, Wang X, Li B, Wang Y, Li Y and Yang L: Network pharmacology-based prediction of the active ingredients and potential targets of Chinese herbal Radix Curcumae formula for application to cardiovascular disease. J Ethnopharmacol 145 : $1-10,2013$.

13. Yue SJ, Liu J, Feng WW, Zhang FL, Chen JX, Xin LT, Peng C Guan HS, Wang CY and Yan D: System pharmacology-based dissection of the synergistic mechanism of huangqi and huanglian for diabetes mellitus. Front Pharmacol 8: 694,2017.

14. Kuhn M, Szklarczyk D, Franceschini A, von Mering C, Jensen LJ and Bork P: STITCH 3: Zooming in on protein-chemical interactions. Nucleic Acids Res 40 (Database issue): D876-D880, 2012.

15. Koscielny G, An P, Carvalho-Silva D, Cham JA, Fumis L, Gasparyan R, Hasan S, Karamanis N, Maguire M, Papa E, et al: Open Targets: A platform for therapeutic target identification and validation. Nucleic Acids Res 45 (D1): D985-D994, 2017.

16. Carvalho-Silva D, Pierleoni A, Pignatelli M, Ong C, Fumis L, Karamanis N, Carmona M, Faulconbridge A, Hercules A, McAuley E, et al: Open targets platform: New developments and updates two years on. Nucleic Acids Res 47 (D1): D1056-D1065, 2019.

17. Wishart DS, Knox C, Guo AC, Shrivastava S, Hassanali M, Stothard P, Chang Z and Woolsey J: DrugBank: A comprehensive resource for in silico drug discovery and exploration. Nucleic Acids Res 34 (Database issue): D668-D672, 2006.

18. Wishart DS, Knox C, Guo AC, Cheng D, Shrivastava S, Tzur D, Gautam B and Hassanali M: DrugBank: A knowledgebase for drugs, drug actions and drug targets. Nucleic Acids Res 36 (Database issue): D901-D906, 2008

19. Wishart DS, Feunang YD, Guo AC, Lo EJ, Marcu A, Grant JR, Sajed T, Johnson D, Li C, Sayeeda Z, et al: DrugBank 5.0: A major update to the DrugBank database for 2018. Nucleic Acids Res 46 (D1): D1074-D1082, 2018.

20. Kafkas S, Dunham I and McEntyre J: Literature evidence in open targets-a target validation platform. J Biomed Semantics 8 20, 2017.

21. Shannon P, Markiel A, Ozier O, Baliga NS, Wang JT, Ramage D, Amin N, Schwikowski B and Ideker T: Cytoscape: A software environment for integrated models of biomolecular interaction networks. Genome Res 13: 2498-2504, 2003.

22. von Mering C, Jensen LJ, Snel B, Hooper SD, Krupp M, Foglierini M, Jouffre N, Huynen MA and Bork P: STRING: Known and predicted protein-protein associations, integrated and transferred across organisms. Nucleic Acids Res 33 (Database issue): D433-D437, 2005.

23. Yu G, Wang LG, Han Y and He QY: ClusterProfiler: An R package for comparing biological themes among gene clusters. OMICS 16: 284-287, 2012.
24. Huntley RP, Harris MA, Alam-Faruque Y, Blake JA, Carbon S, Dietze H, Dimmer EC, Foulger RE, Hill DP, Khodiyar VK, et al: A method for increasing expressivity of Gene Ontology annotations using a compositional approach. BMC Bioinformatics 15: 155,2014

25. Ashburner M, Ball CA, Blake JA, Botstein D, Butler $\mathrm{H}$, Cherry JM, Davis AP, Dolinski K, Dwight SS, Eppig JT, et al: Gene ontology: Tool for the unification of biology. The Gene Ontology Consortium. Nat Genet 25: 25-29, 2000.

26. Ritchie ME, Phipson B, Wu D, Hu Y, Law CW, Shi W and Smyth GK: Limma powers differential expression analyses for RNA-sequencing and microarray studies. Nucleic Acids Res 43: e47, 2015

27. Troyanskaya O, Cantor M, Sherlock G, Brown P, Hastie T, Tibshirani R, Botstein D and Altman RB: Missing value estimation methods for DNA microarrays. Bioinformatics 17: 520-525, 2001.

28. Sanchez-Linares I, Perez-Sanchez H, Cecilia JM and Garcia JM: High-Throughput parallel blind virtual screening using BINDSURF. BMC Bioinformatics 13 Suppl 14 (Suppl 14): S13, 2012.

29. Burley SK, Berman HM, Bhikadiya C, Bi C, Chen L, Di Costanzo L, Christie C, Dalenberg K, Duarte JM, Dutta S, et al: RCSB Protein Data Bank: Biological macromolecular structures enabling research and education in fundamental biology, biomedicine, biotechnology and energy. Nucleic Acids Res 47 (D1): D464-D474, 2019.

30. Science Mo and China TotPsRo: Guidance suggestions for the care and use of laboratory animals. 2006.

31. Li X, Du M, Liu X, Wu M, Ye H, Lin J, Chen W and Wu G: Millimeter wave treatment inhibits NO-induced apoptosis of chondrocytes through the p38MAPK pathway. Int J Mol Med 25: 393-399, 2010.

32. Li XH, Wu MX, Ye HZ, Chen WL, Lin JM, Zheng LP and Liu XX: Experimental study on the suppression of sodium nitroprussiate-induced chondrocyte apoptosis by Tougu Xiaotong Capsule-containing serum. Chin J Integr Med 17: 436-443, 2011

33. Sanchez C, Mathy-Hartert M, Deberg MA, Ficheux H, Reginster JY and Henrotin YE: Effects of rhein on human articular chondrocytes in alginate beads. Biochem Pharmacol 65: 377-388, 2003

34. Wu SQ, Otero M, Unger FM, Goldring MB, Phrutivorapongkul A, Chiari C, Kolb A, Viernstein H and Toegel S: Anti-inflammatory activity of an ethanolic Caesalpinia sappan extract in human chondrocytes and macrophages. J Ethnopharmacol 138: 364-372, 2011.

35. Livak KJ and Schmittgen TD: Analysis of relative gene expression data using real-time quantitative PCR and the 2(-Delta Delta C(T)) method. Methods 25: 402-408, 2001.

36. Lin J, Wu G, Chen J, Fu C, Hong X, Li L, Liu X and Wu M: Electroacupuncture inhibits sodium nitroprusside-mediated chondrocyte apoptosis through the mitochondrial pathway. Mol Med Rep 18: 4922-4930, 2018.

37. Takamatsu Y, Sugiyama A, Purqon A, Nagao H and Nishikawa K: Binding free energy calculation and structural analysis for antigen-antibody complex. J 832: 566-569, 2006.

38. Liao PC, Lai MH, Hsu KP, Kuo YH, Chen J, Tsai MC, Li CX, Yin XJ, Jeyashoke N and Chao LK: Identification of $\beta$-sitosterol as in vitro anti-inflammatory constituent in moringa oleifera. J Agric Food Chem 66: 10748-10759, 2018.

39. Fukai A, Kamekura S, Chikazu D, Nakagawa T, Hirata M, Saito T, Hosaka Y, Ikeda T, Nakamura K, Chung UI and Kawaguchi H: Lack of a chondroprotective effect of cyclooxygenase 2 inhibition in a surgically induced model of osteoarthritis in mice. Arthritis Rheum 64: 198-203, 2012

40. Zhang LC, $\mathrm{Hu} \mathrm{Y}$ and Sui C: Lack of associations between polymorphisms in SOD2 (rs2758331), NOS3 (rs1808593), PPARS (rs9794 and rs10865710) and the risk of osteoarthritis in a Chinese Han population: A case-control study. Chin Med J (Engl) 132: 1113-1114, 2019.

41. Crettol S, Déglon JJ, Besson J, Croquette-Krokkar M, Gothuey I, Hämmig R, Monnat M, Hüttemann H, Baumann P and Eap CB: Methadone enantiomer plasma levels, CYP2B6, CYP2C19, and CYP2C9 genotypes, and response to treatment. Clin Pharmacol Ther 78: 593-604, 2005.

42. Lee CM, Tripathi S and Morgan ET: Nitric oxide-regulated proteolysis of human CYP2B6 via the ubiquitin-proteasome system. Free Radic Biol Med 108: 478-486, 2017.

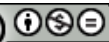

This work is licensed under a Creative Commons Attribution-NonCommercial-NoDerivatives 4.0 International (CC BY-NC-ND 4.0) License. 